\title{
Fully printed 3 microns thick dielectric elastomer actuator
}

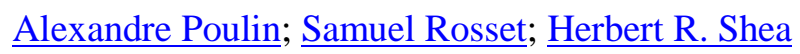

Proc. SPIE 9798, Electroactive Polymer Actuators and Devices (EAPAD) 2016, 97980L

(April 15, 2016); doi:10.1117/12.2218256

Copyright notice format: Copyright 2016 Society of Photo-Optical Instrumentation Engineers. One print or electronic copy may be made for personal use only. Systematic reproduction and distribution, duplication of any material in this paper for a fee or for commercial purposes, or modification of the content of the paper are prohibited. 


\title{
Fully printed 3 microns thick dielectric elastomer actuator
}

\author{
A. Poulin, S. Rosset and H. Shea \\ Microsystems for Space Technologies Laboratory, Ecole Polytechnique Fédérale de Lausanne \\ (EPFL), Neuchâtel, Switzerland
}

\begin{abstract}
In this work we present a new fabrication technique to print thin dielectric elastomer actuators (DEAs), reducing the driving voltage below $300 \mathrm{~V}$ while keeping good actuation performance. With operation voltages in the $\mathrm{kV}$-range, standard DEAs are limited in terms of potential applications. Using thinner membranes is one of the few existing methods to achieve lower operation voltages. Typical DEAs have membranes in the 20-100 $\mu \mathrm{m}$ range, values below which membrane fabrication becomes challenging and the membrane quality and uniformity degrade. Using pad printing we produced thin silicone elastomer membranes, on which we pad-printed compliant electrodes. We then fabricated DEAs by assembling two membranes back to back. We obtain an actuation strain of $7.5 \%$ at only $245 \mathrm{~V}$ on a $3 \mu \mathrm{m}$ thick DEA. In order to investigate the stiffening impact of the electrodes we developed a simple DEA model that includes their mechanical properties. We also developed a strain-mapping algorithm based on optical correlation. The simulation results and the strain-mapping measurements confirm that the stiffening impact of the electrodes increases for thinner membranes. Electrodes are an important element that cannot be neglected in the design and optimization of ultra-thin DEAs.
\end{abstract}

\section{INTRODUCTION}

In this work we present a novel fabrication technique to print thin dielectric elastomer actuators (DEAs), reducing the driving voltage below $300 \mathrm{~V}$ while maintaining good actuation performance. We have recently reported a similar device ${ }^{1}$ and expand here on the fabrication, characterization and modeling aspects of our work. Stretchability, biocompatibility, optical transparency and silent operation are all exciting advantages of DEAs, making this technology an ideal candidate for a wide range of applications ranging from well-established fields such as optics ${ }^{2,3}$ and fluidics ${ }^{4,5}$ to emerging fields such as mechanobiology ${ }^{6}$ and soft-robotics ${ }^{7-9}$. With voltages typically in the $\mathrm{kV}$ range, DEAs are however limited in terms of real-world applications, where cost and size of high-voltage electronics is an important drawback.

For small deformation and ignoring the electrodes stiffness, lateral $s_{x}=s_{y}$ and vertical $s_{z}$ strains can be approximated ${ }^{10}$ by:

$$
s_{x}=-\frac{s_{z}}{2}=\epsilon \frac{E^{2}}{2 Y}=\epsilon \frac{V^{2}}{2 t^{2} Y}
$$

where $\epsilon$ is the dielectric permittivity of the membrane, $Y$ is its Young's modulus, $E$ is the electric field between the two electrodes, $V$ is the applied voltage and $t$ is the membrane thickness. The driving voltage of DEAs can be reduced by optimizing the material properties of the elastomer membrane, or by reducing its thickness. The Young's modulus and dielectric permittivity of elastomers can be engineered ${ }^{11}$ using different techniques such as the addition of plasticizers ${ }^{12}$ and fillers ${ }^{13,14}$. It is only recently that significant improvement was reported over typical DEA materials, synthesizing an elastomer with high dielectric permittivity and good mechanical properties ${ }^{15}$. The reported actuator was however based on a $180 \mu \mathrm{m}$ thick membrane and required $1 \mathrm{kV}$ to reach a lateral strain of 7\%. A different and complementary approach consists in decreasing the elastomer membrane thickness. While typical actuators are $20 \mu \mathrm{m}$ to $200 \mu \mathrm{m}$ in thickness, different techniques can be used to make sub-micron elastomer membranes ${ }^{16}$. It is however very challenging to achieve thickness uniformity and breakdown field compatible for use with DEAs.

We present here the use of pad printing to produce fully printed DEAs with membrane thickness of only a few microns. Pad printing is an industrial printing process mostly used to print inks on three dimensional surfaces otherwise difficult to print on. The technique transfers a pattern from a cliché onto a substrate via a silicone pad. With a clever choice of materials, and by carefully adjusting the printing parameters such as pad pressure and number of stamping steps, we can produce uniform silicone membranes of $1 \mu \mathrm{m}$ to $10 \mu \mathrm{m}$ in thickness. To make a DEA we first pad-printed two elastomer membranes, on top of which we next pad-printed compliant electrodes based on a carbon black-elastomer composite

Electroactive Polymer Actuators and Devices (EAPAD) 2016, edited by Yoseph Bar-Cohen, Proc. of SPIE Vol. 9798, 97980L · C 2016 SPIE · CCC code: 0277-786X/16/\$18 · doi: 10.1117/12.2218256 
ink $^{17}$. We finally assembled the two membranes back to back and applied a $10 \%$ equibiaxial prestretch. The total membrane thickness after prestretch was $3 \mu \mathrm{m}$, ten times thinner than typical DEAs. Using this device we report a lateral actuation strain of $7.5 \%$ at only $245 \mathrm{~V}$. It corresponds to a strain-to-voltage-squared ratio of $125 \% / \mathrm{kV}^{2}$, the highest reported value for DEA to date by more than an order of magnitude.

To investigate the mechanical impact of the $\mu \mathrm{m}$ thick electrodes on the device performance we developed an energybased model that includes the electrodes mechanical properties, a parameter typically neglected for standard DEAs but which should not be overlooked for thin DEAs. We also developed a strain-mapping algorithm based on optical correlation and analyzed the strain profile of our actuator. Results show that the electrode stiffness is indeed a critical parameter, and gives insight on how to design and optimize ultra-thin DEAs.

\section{PAD PRINTING PROCESS FOR DEA FABRICATION}

Ultra-thin DEAs for low voltage applications present significant challenges. The fabrication of high quality membranes that can sustain high electric field is difficult below $20 \mu \mathrm{m}$ where the presence of membrane defects and thickness variations become critical. The fabrication of ultra-thin DEAs has also been limited by the need for a technique to pattern stretchable electrodes on extremely soft membranes without inducing any mechanical damage, and with limited stiffening impact.

We report a fabrication process that overcomes those technical limitations. Our process is based on pad printing, an established technology mostly used in the industry to print 2D ink patterns on three dimensional surfaces. Pad printing machines use a cliché (steel plate engraved with the desired design) whose recessed features are filled by an ink pot sliding on its surface. A silicone pad is pressed against the cliché and then removed, thus picking up the ink from the cliché. The pad is next pressed against the printing substrate and removed, thus transferring the ink from the pad to the substrate $^{17}$. Pad printing uniform layers of high-viscosity materials such as silicone elastomers is not trivial. It is with a clever choice of materials, and by carefully adjusting the printing parameters such as pad pressure and number of stamping steps, that we were able to print uniform silicone membranes of $1 \mu \mathrm{m}$ to $10 \mu \mathrm{m}$ in thickness.

The ultra-thin DEA fabrication process that we developed based on pad printing is detailed in Figure 1.

(i) A substrate of high-quality polyethylene terephthalate (PET) foil was coated with a water soluble sacrificial layer of poly (acrylic) acid (PAA). The sacrificial layer was obtained from a solution of 5\% PAA, $15 \% \mathrm{H}_{2} \mathrm{O}$ and $80 \%$ isopropyl alcohol (IPA) applied by bar coating on a A4 sheet of PET. The substrate was next laser cut into $50 \mathrm{~mm}$ diameter circular pieces.

(ii) Using a pad printing machine (TMP-101, Teca-Print AG, Switzerland) a silicone layer was printed on the substrate. Different silicones such as the Sylgard 184 (Dow Corning, USA), Sylgard 186 (Dow Corning, USA) and Silbione LSR 4305 (Bluestar Silicones, France) were successfully printed. The more viscous silicones required the addition of solvent (OS-2, Dow Corning, USA) which led to lower quality membranes. The best results were obtained with Sylgard 184 for which no solvent was required due to its low viscosity (3500 cP compared to $65000 \mathrm{cP}$ for Sylgard 186 and $40000 \mathrm{cP} 186$ for LSR 4305) and good self-leveling properties. The substrate was allowed $30 \mathrm{~min}$ to self-level after printing, significantly improving the silicone layer uniformity. The substrate was then heat-cured at $80^{\circ} \mathrm{C}$ for $60 \mathrm{~min}$.

(iii) A stretchable electrode was pad printed on the cured silicone layer and heat-cured at $80^{\circ} \mathrm{C}$ for $60 \mathrm{~min}$. We used a carbon black-elastomer composite ink developed in our laboratory ${ }^{17}$ which provides stable mechanical and electrical properties and has a limited stiffening impact. Pad printing could not be used to apply electrodes on suspended ultra-thin membranes i.e. we had to print the electrodes before releasing the membrane and had to minimize the printing pressure of the pad in order to avoid any mechanical damage.

(iv) The sample was immersed in hot deionized water for $5 \mathrm{~min}$, dissolving the PAA sacrificial layer and releasing the printed membrane. With this technique the membrane release was gradual and very gentle on the membrane, avoiding any mechanical damage.

(v) Two released membranes were placed under an equibiaxial prestretch. Different prestretch levels were tested and samples were stretched by more than 2 times their initial dimensions, demonstrating the good quality of the printed membranes. A low prestretch level was nevertheless preferred in order to limit its impact on the electrodes 
resistance (The electrodes are applied before prestretch which therefore influence their resistance). The results presented in this work were obtained under a 1.1 equibiaxial prestretch.

(vi) The two membranes were assembled back-to-back to form a DEA. A drop of ethanol was placed between the two membranes to ensure a uniform contact and avoid trapping air bubbles. Due to the presence of a thin layer of alcohol between them, the two layers could slide on top of each other, which enabled precise electrodes alignment. After $5 \mathrm{~min}$ at room temperature the alcohol was completely evaporated and surface adhesion held the two membranes together. Poly (methyl methacrylate) (PMMA) rings were fixed on both sides of the actuator using pressure sensitive adhesive (ARclear, Adhesive Research Inc.) to lock the membrane prestretch.

(i)

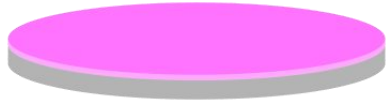

(ii)
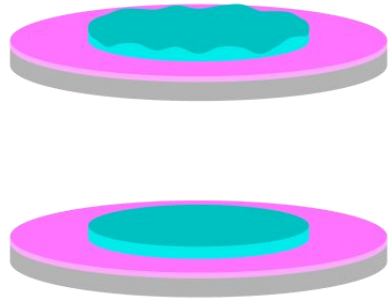

(iii)
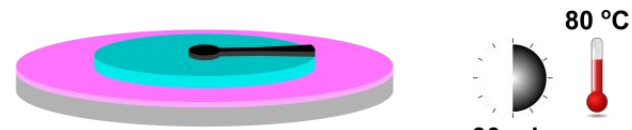

$30 \mathrm{~min}$
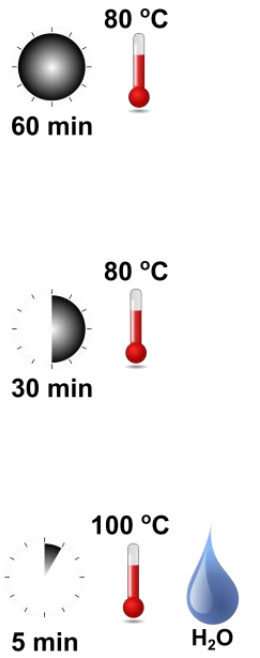

(iv)

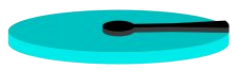

$5 \mathrm{~min}$ (v)

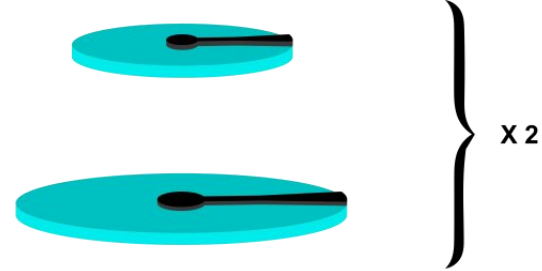

(vi)
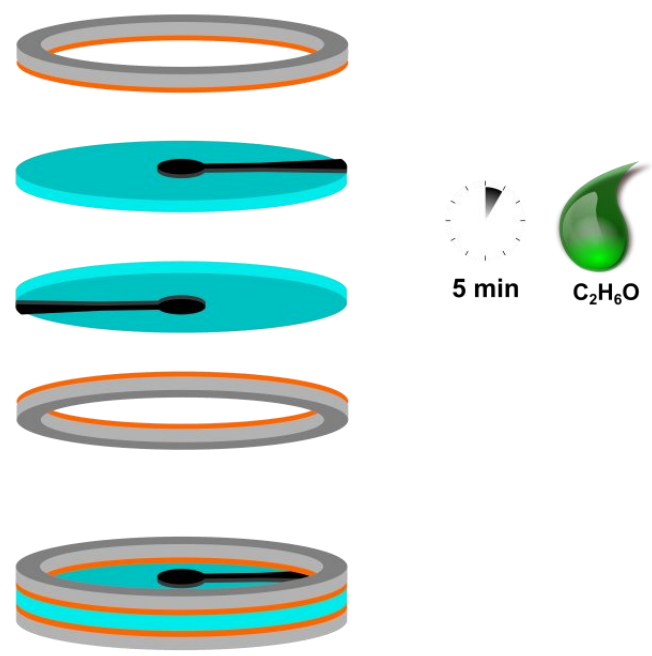

Figure 1: Pad printing was used to produce fully printed DEAs. (i) A water soluble sacrificial layer of PAA was applied on a PET foil. (ii) A silicone layer was then transferred on the substrate using a pad printing machine. The printed silicone layer was allowed time to self-level before it was heat-cured. (iii) Using the same pad printing machine we printed a stretchable electrode on the cured silicone membrane. The carbon-elastomer composite electrode was then heat-cured. (iv) The printed membrane was immersed in hot water to dissolve the sacrificial layer, releasing it from the PET substrate. (v) Two membranes were printed and placed under equibiaxial prestretch. (vi) The two membranes were then assembled back-toback to form a DEA, using ethanol to ensure a uniform contact between the membranes and avoid trapping air bubbles. PMMA rings were fixed on both sides of the actuator using pressure sensitive to lock the membrane prestretch. 


\section{ACTUATION PERFORMANCE OF PRINTED DEAS}

Using the fabrication process presented in the previous section we fabricated a fully printed $3 \mu \mathrm{m}$ thick DEA and evaluated its performance, comparing it with a typical $30 \mu \mathrm{m}$ thick DEA that was fabricated following our standard fabrication process ${ }^{17}$. We used expanding circle actuators for this study, a standard for performance evaluation of DEAs $^{18}$. Except for the different membrane thicknesses, the $3 \mu \mathrm{m}$ and $30 \mu \mathrm{m}$ devices were identical. They had the same geometry, used the same membrane and electrode materials, and had the same membrane prestretch. Figure 2 (a) presents a picture of the $3 \mu \mathrm{m}$ printed DEA where the stretchable electrodes appear in black. The electrodes were $1 \mu \mathrm{m}$ to $2 \mu \mathrm{m}$ in thickness, slightly thinner than the printed DEA membrane. The circular active area was $2 \mathrm{~mm}$ in diameter and the rigid PMMA rings holding the membrane prestretch had an inner diameter of $20 \mathrm{~mm}$. The membrane was made of Sylgard 184 and placed under a 1.1 equibiaxial prestretch.

Figure 2 (b) presents the average radial strain of the $3 \mu \mathrm{m}$ and $30 \mu \mathrm{m}$ DEAs as a function of the driving voltage. The actuation on the thin membrane was limited by dielectric breakdown and a maximum strain of $7.5 \%$ was achieved at only $245 \mathrm{~V}$. For comparison, actuation on the thick membrane required $3.3 \mathrm{kV}$ to reach the same strain level. Limited by loss of mechanical tension, actuation on the thick membrane could reach up to $14.2 \%$ strain at $4.1 \mathrm{kV}$. Overall, the $3 \mu \mathrm{m}$ printed DEA was limited to half the strain of its $30 \mu \mathrm{m}$ thick equivalent. This downside was however easily offset by a more than ten-fold decrease of the driving voltage. In addition, a radial strain of $7.5 \%$ is enough to meet the requirements of various DEA applications ${ }^{19,20}$.

The limited maximum strain of the printed DEA can have two main origins. a) The presence of non-uniformities in the membrane such as thickness variations, air bubbles or inclusion of foreign particles that can lead to premature dielectric breakdown. b) The mechanical stiffening impact of the electrodes which can increase the electric field required to reach a given strain level, effectively decreasing the strain at dielectric breakdown. It is generally difficult to improve membrane uniformities and to reduce electrodes stiffness as those parameters are often already optimized. Further improvement on the electrode stiffness for example would come at the cost of higher resistance. As a result, their relative impact on DEA performance typically increases as the membrane thickness decreases.

A good comparison metric to evaluate this work is the strain-to-voltage-squared ratio $\left(\mathrm{S}_{\mathrm{x}} / \mathrm{V}^{2}\right)$ which, unlike the more intuitive strain-to-voltage ratio $\left(\mathrm{S}_{\mathrm{x}} / \mathrm{V}\right)$, does not depend on the actuation parameters but solely on the device geometry. Equation 1 clearly shows that $S_{x} / V^{2}$ is a device-constant parameter while $S_{x} / V$ varies linearly with $V$. Using this metric as a factor of merit the printed DEA improved the actuator performance by more than two orders of magnitude, increasing the $S_{x} / \mathrm{V}^{2}$ ratio from $0.7 \% / \mathrm{kV}^{2}$ for the $30 \mu \mathrm{m}$ thick device to $125 \% / \mathrm{kV}^{2}$ for the $3 \mu \mathrm{m}$ thick device. This result is the highest reported value for a DEA, improving by more than one order of magnitude over current state-of-the art devices $^{1}$. While area strain of $488 \%{ }^{21}$ and actuation at only $10 \mathrm{~V} / \mu \mathrm{m}^{15}$ have been reported for expanding circle DEAs, the corresponding strain-to-voltage-squared ratios were only $5.7 \% / \mathrm{kV}^{2}$ and $7 \% / \mathrm{kV}^{2}$ respectively.
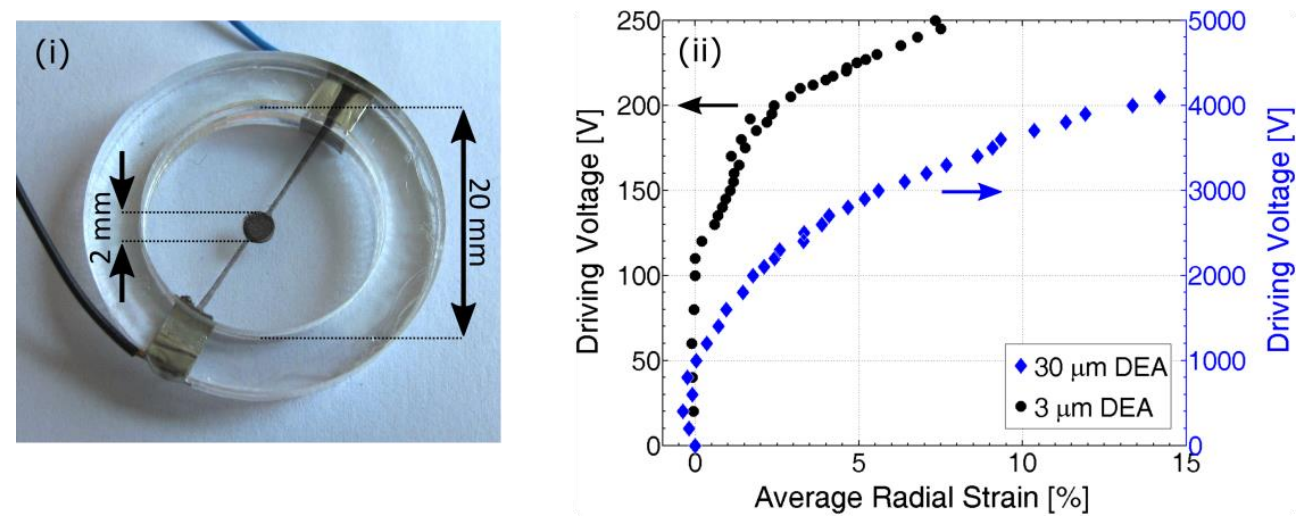

Figure 2: (i) Picture of the printed $3 \mu \mathrm{m}$ thick DEA with a $2 \mathrm{~mm}$ diameter active area on a $20 \mathrm{~mm}$ diameter membrane. (ii) The average radial strain was measured on $3 \mu \mathrm{m}$ and $30 \mu \mathrm{m}$ thick DEAs. Except for the different membrane thicknesses, both actuators had identical geometry, materials and prestretch. The printed $3 \mu \mathrm{m}$ thick DEA was limited to lower actuation strain but it required more than 10 times lower driving voltage. It exhibited $7.5 \%$ radial strain at only $245 \mathrm{~V}$, which corresponds to a strain-to-voltage squared ratio of $125 \% / \mathrm{kV}^{2}$. 


\section{STRAIN FIELD UNIFORMITY OF PRINTED DEAS}

The actuation strain of DEAs is typically measured by tracking the electrodes boundary and calculating the ratio between their initial and final dimensions. This approach is quick and easy to implement but it can hide some valuable information. The ratio between the initial and final dimensions of the electrodes gives the average strain but it provides no information on how uniform the strain field is. In order to get a better insight on the actuation performance of the printed DEA we characterized the uniformity of its strain field.

In addition to the technical difficulty and complexity of measuring strain over a large array of points (instead of only tracking one or two boundaries for average strain), the main difficulty in mapping strain fields comes from the electrode material. Usually highly absorbent in the visible range, it is very challenging to make optical measurements in the electrode-covered regions. The use of periodic metallic microstructures ${ }^{22}$ and randomly dispersed fluorescent microbeads $^{23}$ can be used to overcome this limitation. Those two techniques however require very specialized microfabrication or imaging equipment.

In this work we removed the need for metallic microstructure or fluorescent markers by taking advantage of the electrodes microstructure. Figure 3 summarises the mains steps of the strain mapping process.

(i) The DEA was placed under a microscope and pictures of the device were recorded under increasing driving voltages. We adjusted the sample lighting to maximise contrast and avoid saturation of the camera sensor in the electrode-coated regions.

(ii) The unactuated picture was discretized into an array of element $\left(e_{x}, e_{y}\right)$ each composed of $n_{x}$ by $n_{y}$ pixels with a center point located at the pixel coordinate $\left(\mathrm{p}_{\mathrm{x}}, \mathrm{p}_{\mathrm{y}}\right)$. Using normalized cross-correlation, a technique widely used for pattern recognition, we found for each element their new position $\left(\mathrm{p}_{\mathrm{x}}{ }^{\prime}, \mathrm{p}_{\mathrm{y}}{ }^{\prime}\right)$ in the actuated picture.

The cross-correlation step requires the element to be scanned over every pixel of the actuated picture, calculating for each position a correlation coefficient $C$. The coefficient can take any value between -1 and 1 (perfect match), and the final output is a 3D surface with its maximum $C_{\max }$ located at the best match position $\left(\mathrm{p}_{\mathrm{x}}{ }^{\prime}, \mathrm{p}_{\mathrm{y}}{ }^{\prime}\right)$. If $C_{\max }$ is greater than a predefined confidence threshold, we have found the new element position $\left(p_{x}{ }^{\prime}, p_{y}{ }^{\prime}\right)$. To minimize processing time the cross-correlation can be calculated over a limited region of the actuated picture, for example a region centered on $\left(\mathrm{p}_{\mathrm{x}}, \mathrm{p}_{\mathrm{y}}\right)$ and measuring $\mathrm{n}_{\mathrm{y}}+2 \delta_{\mathrm{y}}$ by $\mathrm{n}_{\mathrm{x}}+2 \delta_{\mathrm{x}}$ pixels can be used.

Strain levels greater than $5 \%$ can lead to poor results when using a normalized cross-correlation algorithm. One solution is to digitally stretch the elements before the cross-correlation step. This approach however requires guessing the expected strain and can require long iteration loops in order to reach good correlation coefficients. A second approach is to simply process a series of pictures recorded at intermediate steps, adding up the strain maps to obtain the total strain field.

(iii) Finally, from the initial $\left(p_{x}, p_{y}\right)$ and final positions $\left(p_{x}{ }^{\prime}, p_{y}{ }^{\prime}\right)$ we calculated the displacement and strain fields. The displacement field was simply obtained by calculating the distance separating the two positions. The overlaid black arrows in Figure 3 (iii) correspond to the displacement direction. The amplitude of the displacement is given by the arrows length and by the color coded contour lines. The strain field was obtained by calculating the derivative of the displacement field. The overlaid black lines in Figure 3 (iii) correspond to the vertical/horizontal strain ratio i.e. $45^{\circ}$ lines indicates an equibiaxial strain, horizontal lines correspond to a uniaxial horizontal strain, and vertical lines correspond to a uniaxial vertical strain. The strain amplitude is given by the lines length and by the color coded contour lines.

Strain mapping showed excellent uniformity for the $30 \mu \mathrm{m}$ DEA but showed noticeable variations for the $3 \mu \mathrm{m}$ DEA. Figure 3 (iii) presents the $3 \mu \mathrm{m}$ DEA strain field, and whereas the average lateral strain was only $7.5 \%$, local strain values greater than $10 \%$ were measured in some area of the device. This result gives an important insight on the actuation performance of the printed DEA i.e. Regions under higher strain are exposed to higher electric field and are consequently prone to induce early dielectric breakdown. Actuation strain of the printed DEA was therefore partially limited by its non-uniform strain profile.

The non-uniform strain profile measured on the $3 \mu \mathrm{m}$ thick DEA can origin from thickness variations over the membrane and from the electrodes stiffening impact (i.e. the effects of electrodes thickness variations and the presence of leads connecting the circular active area). While both effects must have influenced the device performance, we believe that the electrodes mechanical impact was dominating. White light interferometry measurements showed good membrane 
thickness uniformity with less than $10 \%$ variations, which according to our simulation is not enough to explain the variations measured in the strain profile. In addition, the same strain distribution presented in Figure 3 was observed on all printed DEAs i.e. a high strain region located in the center of the device, and elongated in the direction perpendicular to the feedlines. This regular strain profile can be explained by the electrodes mechanical impact but not by random thickness variations over the membrane.

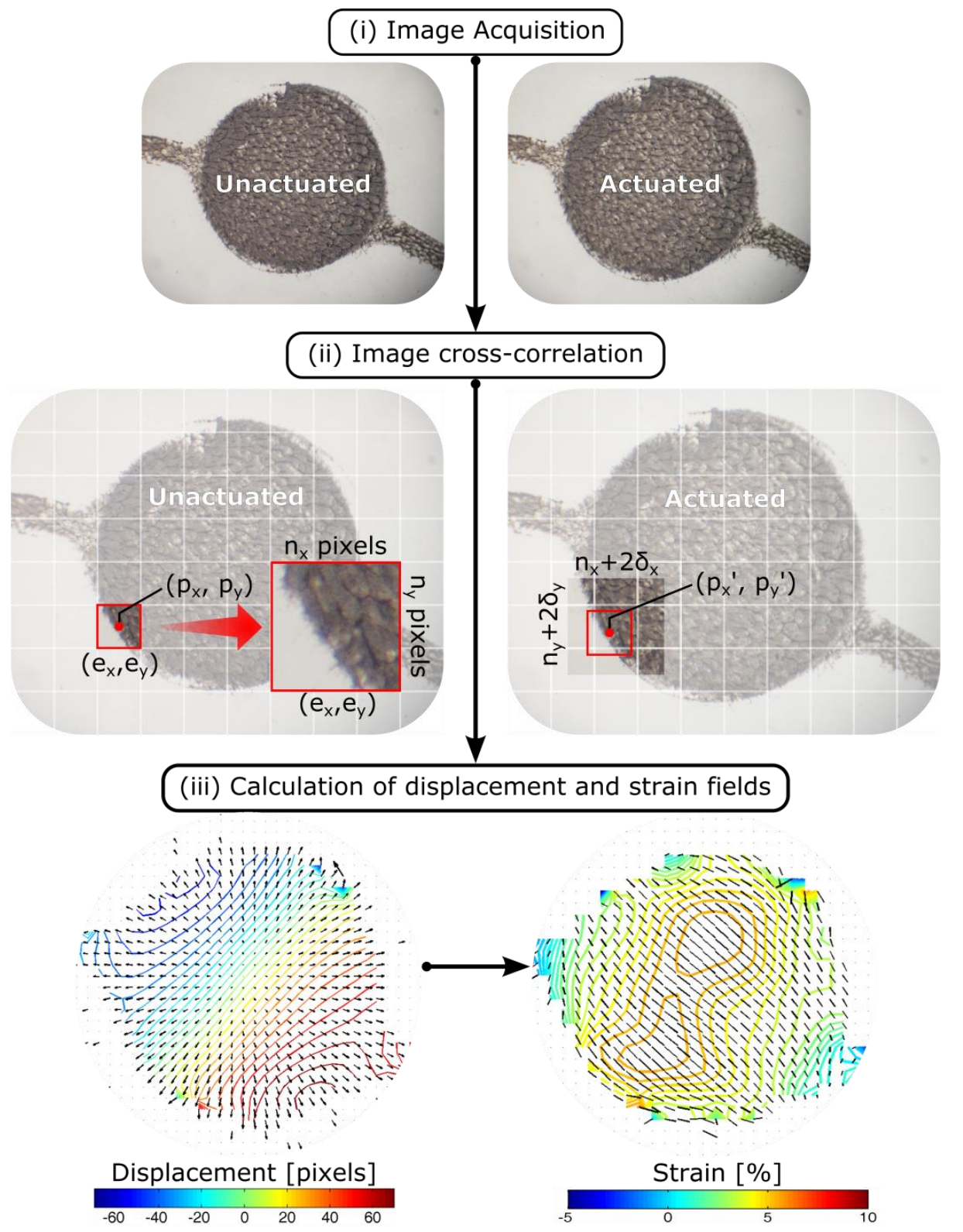

Figure 3: Digital Image Correlation (DIC) was used to measure the strain field of the actuator. Taking advantage of the electrodes topography we achieve this measurement without the need for additional markers as typically required for DIC. (i) The fully printed DEA was actuated while pictures of the active area were recorded at different voltages. (ii) The unactuated picture was first discretized into an array of elements $\left(\mathrm{e}_{\mathrm{x}}, \mathrm{e}_{\mathrm{y}}\right)$, each composed of $\mathrm{n}_{\mathrm{x}}$ by $\mathrm{n}_{\mathrm{y}}$ pixels and with their center points located at the pixels coordinates $\left(\mathrm{p}_{\mathrm{x}}, \mathrm{p}_{\mathrm{y}}\right)$. Using normalized cross-correlation, a technique widely used for pattern recognition, we found for each element its new position $\left(\mathrm{p}_{\mathrm{x}}{ }^{\prime}, \mathrm{p}_{\mathrm{y}}{ }^{\prime}\right)$ in the actuated picture. To minimize processing time we calculated correlation only over a sub-region centered on $\left(\mathrm{p}_{\mathrm{x}}, \mathrm{p}_{\mathrm{y}}\right)$ and measuring $\mathrm{n}_{\mathrm{y}}+2 \delta_{\mathrm{y}}$ by $\mathrm{n}_{\mathrm{x}}+2 \delta_{\mathrm{x}}$ pixels. (iii) From the position of each element in the unactuated and actuated states we calculated the displacement and strain fields. The overlaid black arrows give the displacement directions while the black line corresponds to the vertical/horizontal strains ratio i.e. a $45^{\circ}$ line indicates equibiaxial strain. 


\section{MODELING THE STIFFENING IMPACT OF ELECTRODES}

Significant work has already been done to develop theoretical models describing the actuation mechanism of DEAs. These models helped identifying and predicting the main failure mechanisms ${ }^{24}$, and were used to maximize DEAs actuation strain ${ }^{21,25}$ and displacement ${ }^{26}$. While the mechanical forces acting in the membrane are usually well described with those models, taking into account the hyperelastic properties and the incompressibility of the material, the mechanics of the electrodes is typically neglected. This approximation is valid for very soft electrodes on thick membranes, but our experimental results on the $3 \mu \mathrm{m}$ DEAs suggest that it is not valid anymore for ultra-thin DEAs.

We developed a simple energy-based model that predicts the actuation strain of an expanding circle DEA as a function of the applied voltage. The boundary effect on the membrane is neglected (a valid approximation if the radius of the active area is much larger than the radius of the passive area) and the prestretch is modeled as a constant force. Figure 4 presents the actuator geometry and lists the different input parameters (in red), variables (in blue) and assumptions (in green) of the model. Using an energy minimization approach we calculated the voltage $V$ as a function of the actuation stretch $\lambda_{\text {act }}$. We first wrote the equations of the different energy contributions, as well as their first derivatives. Using those equations we calculated the total energy of the system and minimized it to find a stable solution. We expressed the analytical solution as a function of the form $V\left(\lambda_{a c t}\right)$ which can be used to predict the voltage required to reach any stretch level for a given actuator design.

Prestretch $\lambda_{\text {pre }}$ was modeled a constant force $F$ acting on the periphery of the stretched layer. The stored mechanical energy can be expressed as

$$
\begin{aligned}
U_{\text {pre }} & =F \delta r \\
& =2 \pi r\left(\lambda_{\text {pre }}\right) t\left(\lambda_{\text {pre }}\right) \sigma\left(\lambda_{\text {pre }}\right)\left(r(\lambda)-r_{0}\right) \\
& =2 \pi r_{0} t_{0}^{2} \lambda_{\text {pre }}^{-1} \sigma\left(\lambda_{\text {pre }}\right)(\lambda-1)
\end{aligned}
$$

where $\lambda=\lambda_{\text {pre }} \lambda_{\text {act }}$ is the total stretch, $r(\lambda)$ is the actuator radius, $t(\lambda)$ is the layer thickness, and $\sigma(\lambda)$ is the mechanical stress in the layer. We replaced $\sigma(\lambda)$ by the Gent model equation for equibiaxial stress and obtained

$$
U_{\text {pre }}=\left[2 \pi r_{0} t_{0}^{2} \lambda_{\text {pre }}^{-1}\left(\lambda_{\text {pre }}-\frac{1}{\lambda_{\text {pre }}^{4}}\right)\left(\frac{\mu J_{m}}{J_{m}-I_{1}\left(\lambda_{\text {pre }}\right)+1}\right)\right](\lambda-1)
$$

and

$$
\frac{d U_{\text {pre }}}{d \lambda_{\text {act }}}=\left[2 \pi r_{0} t_{0}^{2}\left(\lambda_{\text {pre }}-\frac{1}{\lambda_{\text {pre }}^{4}}\right)\left(\frac{\mu J_{m}}{U_{m}-I_{1}\left(\lambda_{\text {pre }}\right)+1}\right)\right]
$$

where $\mu$ is the layer shear modulus, $J_{m}$ is a model parameter and $I_{1}$ is the first invariant of the Cauchy-Green tensor. This equation was used to separately calculate the membrane and electrodes prestretch energy. For the actuation stretch contribution we used the strain energy density function $W$ of the Gent model and obtained

$$
U_{a c t}=-\frac{\mu J_{m}}{2} \ln \left(1-\frac{I_{1}(\lambda)-3}{J_{m}}\right) \pi r_{0}^{2} t_{0}
$$

and

$$
\frac{d U_{a c t}}{d \lambda_{\text {act }}}=\frac{2 \mu J_{m} \pi r_{0}^{2} t_{0}}{\left(J_{m}-I_{1}(\lambda)+3\right)}\left(\frac{\lambda^{6}-1}{\lambda_{a c t} \lambda^{4}}\right)
$$

This equation was used to separately compute the membrane and electrodes actuation stretch energy. For the electrostatic contribution to the total energy we considered a parallel plate capacitor and neglected the edge effect. We obtained

$$
U_{\text {elec }}=\frac{\varepsilon \pi r_{0} \lambda^{4} V^{2}}{2 t_{0}}
$$


and

$$
\frac{d U_{\text {elec }}}{d \lambda_{\text {act }}}=\frac{2 \varepsilon \pi r_{0} \lambda^{3} V^{2}}{t_{0}}
$$

where $\varepsilon$ is the membrane dielectric permittivity and $V$ is the voltage. The total system energy was expressed as

$$
U_{\text {tot }}=2 U_{\text {pre-e }}+U_{\text {pre-m }}+2 U_{\text {act }-e}+U_{\text {act }-m}+U_{\text {elec }}
$$

and setting its first derivative equal to zero (to minimize the system energy) we obtained

$$
-\frac{d U_{\text {elec }}}{d \lambda_{\text {act }}}=2 \frac{d U_{\text {pre }-e}}{d \lambda_{\text {act }}}+\frac{d U_{\text {pre-m }}}{d \lambda_{\text {act }}}+2 \frac{d U_{\text {act }-e}}{d \lambda_{\text {act }}}+\frac{d U_{\text {act }-m}}{d \lambda_{\text {act }}}
$$

where $U_{\text {pre-e }}$ is the prestretch energy of the electrode (In our fabrication process the electrodes were printed before applying prestretch to the membrane), $U_{\text {pre }-m}$ the prestretch energy of the membrane, $U_{\text {act-e }}$ the actuation stretch energy of the electrode, $U_{a c t-m}$ the actuation stretch energy of the membrane, and $U_{\text {elec }}$ the electrostatic energy of the system. Combining Equations 2-5 we isolated the voltage $V$ as a function of the actuation stretch $\lambda_{\text {act }}$ and used this analytical solution to predict the strain-voltage curves for different DEA geometries.

Figure 5 compares the strain-voltage curves of actuators with membrane thicknesses ranging from $5 \mu \mathrm{m}$ to $60 \mu \mathrm{m}$. The solid lines correspond to the results obtained for realistic electrodes of finite stiffness, whereas doted lines corresponds to the results obtained for ideal electrodes of zero stiffness. The red crosses indicate where the electric field in the membrane exceeds the dielectric breakdown strength that was set to $160 \mathrm{~V} / \mu \mathrm{m}$. We considered $1 \mu \mathrm{m}$ thick electrodes that had the same shear modulus as the membrane. The complete list of model parameters is detailed on the right hand side of the figure. As expected, we observed for ideal and realistic electrodes that the driving voltage decreases with the membrane thickness. The strain at dielectric breakdown however evolved differently for the two configurations. It remained constant for the ideal electrodes, but decreased with the membrane thickness for the realistic electrodes.

The offset observed between the two set of curves on Figure 5 comes from the stiffening impact of the electrodes which slightly increases the driving voltage. We observed that the relative stiffening impact of the electrodes is not constant, but that it rather increases as the membrane thickness decreases. This effect is clearly visible in Figure 6 which presents the driving voltage at $5 \%$ radial strain as a function of the membrane thickness. We considered $1 \mu \mathrm{m}$ thick electrodes (constant parameter that was not scaled with the membrane thickness) that had the same shear modulus as the membrane. The complete list of model parameters is detailed on the right hand side of the figure. We observed that the realistic and ideal electrodes models diverge below $15 \mu \mathrm{m}$ thick membranes. As the membrane thickness decreases, the actuator mechanics is gradually dominated by the electrodes rather than the membrane.

The results presented in this section demonstrated that the development of ultra-thin DEAs is not bounded to simply reducing the membrane thickness. We identified the electrodes stiffness as a critical parameter that can lead to premature dielectric breakdown if not carefully considered in the design of the actuator. The simulations results showed that the ideal electrode assumption is not valid anymore for ultra-thin DEAs, or more precisely when the actuator stiffness is not anymore dominated by the mechanical properties of the membrane. Based on those results and on the strain fields measurements presented in the previous section, we believe that the actuation performance of the $3 \mu \mathrm{m}$ DEA was mainly limited by the stiffening impact of the electrodes. One approach to further improve its performance would therefore be to develop a softer and more conductive electrode material that could be applied in thinner layers. 

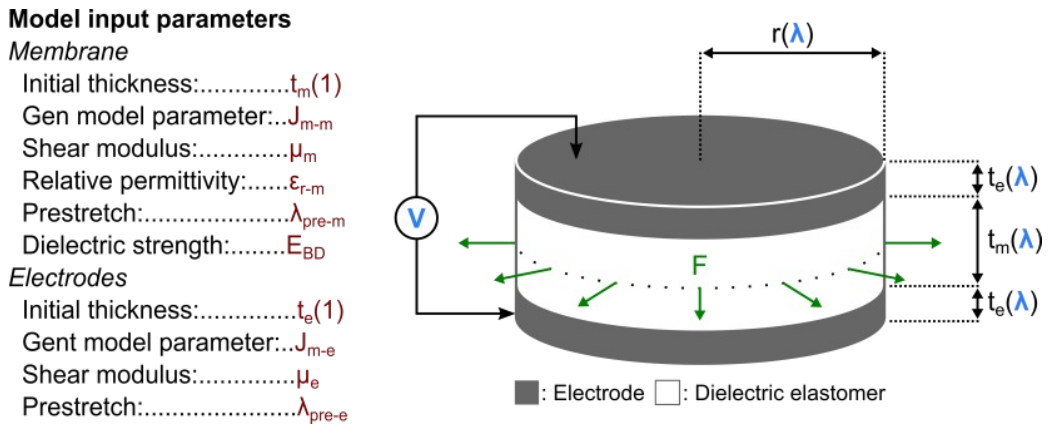

Figure 4: We developed an energy based model to predict the strain of an expanding circle DEA as a function of the applied voltage. It has the particularity of including the electrodes mechanical properties, a critical element in the development of ultra-thin DEAs. The model describes prestretch as a constant force acting at the boundary of the device active area. The mechanical energy stored in the membrane and the electrodes is calculated using the strain energy density function of the Gent model. The figure lists the model assumptions (green), input parameters (red) and variables (blue).
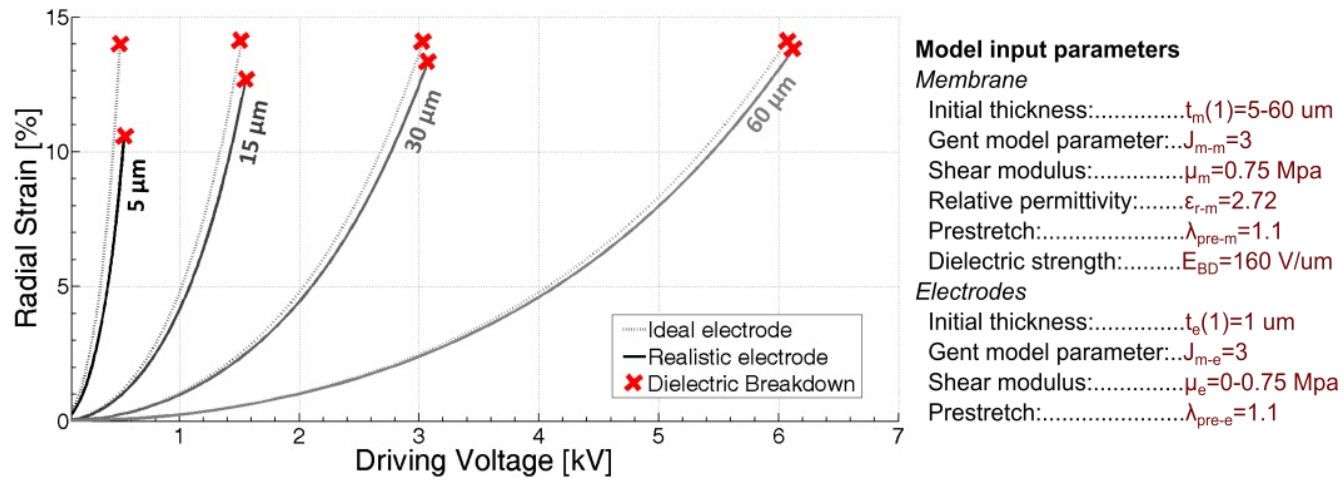

Figure 5: Simulation results for a circular DEA with membrane thicknesses ranging from $5 \mu \mathrm{m}$ to $60 \mu \mathrm{m}$. The solid lines correspond to the results obtained for realistic electrodes of finite stiffness, whereas doted lines corresponds to the results obtained for ideal electrodes of zero stiffness. The red crosses indicate where the electric field in the membrane exceeds the dielectric breakdown strength of the material. The model parameters are listed on the right hand side of the figure. We observed that the realistic electrode model predicts higher actuation voltage due to the mechanical stiffening impact of the electrodes. This effect leads to lower strain at dielectric breakdown, demonstrating that there is an optimal membrane thickness that depends on the DEA voltage and strain requirements.

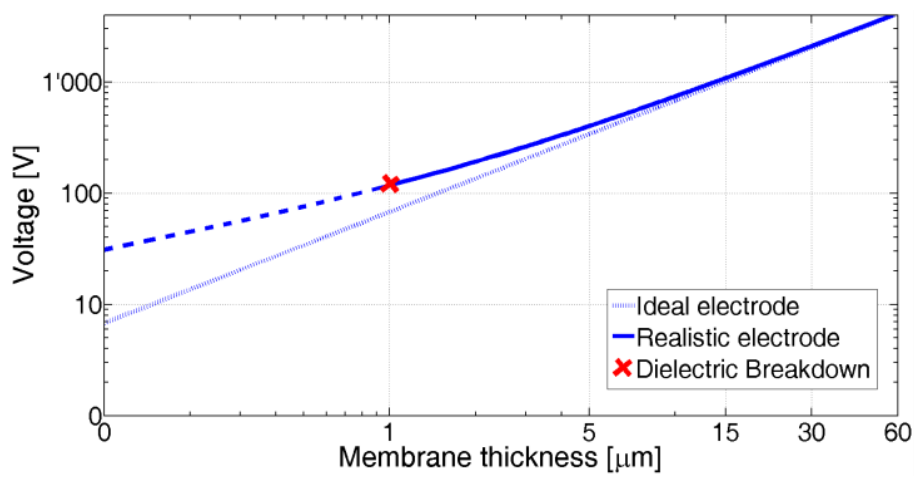

\section{Model input parameters}

Membrane

Initial thickness:................ $\mathrm{t}_{\mathrm{m}}(1)=0-60 \mathrm{um}$

Gent model parameter:... $\mathrm{J}_{\mathrm{m}-\mathrm{m}}=3$

Shear modulus:................ $\mu_{\mathrm{m}}=0.75 \mathrm{Mpa}$

Relative permittivity:........ $\varepsilon_{\mathrm{r}-\mathrm{m}}=2.72$

Prestretch:........................ $\lambda_{\text {pre-m }}=1.1$

Dielectric strength:.......... $\mathrm{E}_{\mathrm{BD}}=160 \mathrm{~V} / \mathrm{um}$

Electrodes

Initial thickness:................. $\mathrm{t}_{\mathrm{e}}(1)=1 \mathrm{um}$

Gent model parameter:... $J_{m-e}=3$

Shear modulus:................. $\mu_{\mathrm{e}}=0-0.75 \mathrm{Mpa}$

Prestretch:...................... $\lambda_{\text {pre-e }}=1.1$

Figure 6: The driving voltage required to reach 5\% radial strain on a circular DEA was calculated and presented here as a function of the membrane thickness. The solid lines correspond to the results obtained for realistic electrodes of finite stiffness, whereas doted lines corresponds to the results obtained for ideal electrodes of zero stiffness. The red crosses indicate where the electric field in the membrane exceeds the dielectric breakdown strength of the material. The model parameters are detailed on the right hand side of the figure. As we decreased the membrane thickness, the actuator stiffness was slowly dominated by the electrodes. As a result we observed that the realistic model slowly diverged from the ideal case, demonstrating that the ideal electrode assumption is not valid for ultra-thin DEAs. 


\section{CONCLUSION}

In this work we presented a fully printed $3 \mu \mathrm{m}$ thick DEA, and demonstrated that decreasing the membrane thickness to only a few microns significantly reduces the driving voltage while maintaining good actuation strain performance. A radial actuation strain of $7.5 \%$ was reported at only $245 \mathrm{~V}$. This result corresponds to an actuation of $125 \% / \mathrm{kV}^{2}$ which is the highest reported value to date for a DEA. In order to investigate the stiffening impact of the electrodes we developed a simple DEA model that includes their mechanical properties. We also developed a strain-mapping algorithm based on optical correlation. The simulation results and the strain-mapping measurements identify the electrodes as an important parameter that should not be neglected in the design and optimization of thin DEAs. This work presents a practical approach toward low-voltage DEAs, a critical step in the development of real-world application. With driving voltage below $300 \mathrm{~V}$, a range covered by commercially available CMOS circuitry, consumer market applications are already possible. Combined with progress in material engineering of elastomers, this work could lead to even lower actuation voltages and open the door to a variety of new opportunities for DEA-based technologies.

\section{ACKNOWLEDGMENTS}

This work was supported by the Swiss National Science Foundation (SNSF) under the grant No. 200020_153122 and the SNSF R'equip program 206021_139187.

\section{REFERENCES}

[1] Poulin, A., Rosset, S.., Shea, H. R., "Printing low-voltage dielectric elastomer actuators," Appl. Phys. Lett. 107(24) (2015).

[2] Carpi, F., Frediani, G., Turco, S.., De Rossi, D., "Bioinspired Tunable Lens with Muscle-Like Electroactive Elastomers," Adv. Funct. Mater. 21(21), 4152-4158 (2011).

[3] Aschwanden, M.., Stemmer, A., "Polymeric, electrically tunable diffraction grating based on artificial muscles," Opt. Lett. 31(17), 2610-2612 (2006).

[4] Xia, F., Tadigadapa, S.., Zhang, Q. M., "Electroactive polymer based microfluidic pump," Sensors Actuators, A Phys. 125(2), 346-352 (2006).

[5] Murray, C., McCoul, D., Sollier, E., Ruggiero, T., Niu, X., Pei, Q.., Carlo, D. Di., “Electro-adaptive microfluidics for active tuning of channel geometry using polymer actuators," Microfluid. Nanofluidics 14(1-2), 345-358 (2013).

[6] Akbari, S.., Shea, H. R., "An array of $100 \mu \mathrm{m} \times 100 \mu \mathrm{m}$ dielectric elastomer actuators with $80 \%$ strain for tissue engineering applications," Sensors Actuators A Phys. 186, 236-241 (2012).

[7] Anderson, I. a., Gisby, T. a., McKay, T. G., O’Brien, B. M.., Calius, E. P., "Multi-functional dielectric elastomer artificial muscles for soft and smart machines," J. Appl. Phys. 112(4), 041101 (2012).

[8] Shintake, J., Rosset, S., Schubert, B., Floreano, D.., Shea, H., "Versatile Soft Grippers with Intrinsic Electroadhesion Based on Multifunctional Polymer Actuators," Adv. Mater. 28(2), 1-8 (2016).

[9] Shian, S., Bertoldi, K.., Clarke, D. R., "Dielectric Elastomer Based ' Grippers ' for Soft Robotics," Adv. Funct. Mater. 27(July), 6814-6819 (2015).

[10] Carpi, F., De Rossi, D., Kornbush, R., Pelrine, R. E.., Sommer-Larson, P., Dielectric Elastomer as Electromechanical Transducers: Fundamentals, Materials, Devices, Models and Applications of an Emerging Electroactive Polymer Technology, Elsevier (2011).

[11] Madsen, F. B., Daugaard, A. E., Hvilsted, S.., Skov, A. L., "The Current State of Silicone-Based Dielectric Elastomer Transducers" (2016).

[12] Niu, X., Stoyanov, H., Hu, W., Leo, R., Brochu, P.., Pei, Q., "Synthesizing a new dielectric elastomer exhibiting large actuation strain and suppressed electromechanical instability without prestretching," J. Polym. Sci. Part B Polym. Phys. 51(3), 197-206 (2013). 
[13] Risse, S., Kussmaul, B., Krüger, H.., Kofod, G., "Synergistic improvement of actuation properties with compatibilized high permittivity filler," Adv. Funct. Mater. 22(18), 3958-3962 (2012).

[14] Romasanta, L. J., Leret, P., Casaban, L., Hernández, M., de la Rubia, M. a., Fernández, J. F., Kenny, J. M., Lopez-Manchado, M. a.., Verdejo, R., "Towards materials with enhanced electro-mechanical response: CaCu3Ti4O12-polydimethylsiloxane composites," J. Mater. Chem., 24705-24712 (2012).

[15] Dünki, S. J., Ko, Y. S., Nüesch, F. a.., Opris, D. M., "Self-Repairable, High Permittivity Dielectric Elastomers with Large Actuation Strains at Low Electric Fields," Adv. Funct. Mater. 25(16), 2467-2475 (2015).

[16] Thangawng, A. L., Ruoff, R. S., Swartz, M. a., Glucksberg, M. R., "An ultra-thin PDMS membrane as a bio/micro-nano interface: fabrication and characterization.," Biomed. Microdevices 9(4), 587-595 (2007).

[17] Rosset, S., Araromi, S., Schlatter, S.., Shea, H., "Fabrication process of silicone-based dielectric elastomer actuators," J. Vis. Exp. 108, 231-238 (2016).

[18] Pelrine, R., "High-Speed Electrically Actuated Elastomers with Strain Greater Than 100\%," Science (80-. ). 287(5454), 836-839 (2000).

[19] Maffli, L., Rosset, S., Ghilardi, M., Carpi, F.., Shea, H., "Ultrafast All-Polymer Electrically Tunable Silicone Lenses," Adv. Funct. Mater. 25(11), 1656-1665 (2015).

[20] Lotz, P., Matysek, M.., Schlaak, H. F., "Fabrication and Application of Miniaturized Dielectric Elastomer Stack Actuators," IEEE/ASME Trans. Mechatronics 16(1), 58-66 (2011).

[21] Huang, J., Li, T., Chiang Foo, C., Zhu, J., Clarke, D. R.., Suo, Z., "Giant, voltage-actuated deformation of a dielectric elastomer under dead load," Appl. Phys. Lett. 100(4) (2012).

[22] Akbari, S.., Shea, H. R., "Microfabrication and characterization of an array of dielectric elastomer actuators generating uniaxial strain to stretch individual cells," J. Micromechanics Microengineering 22(4), 045020 (2012).

[23] Butler, J. P., Tolic, I. V. A. M., Fabry, B. E. N.., Fredberg, J. J., "Traction fields, moments, and strain energy that cells exert on their surroundings '," 595-605 (2002).

[24] Koh, S. J. A., Li, T., Zhou, J., Zhao, X., Hong, W., Zhu, J., Suo, Z., "Mechanisms of large actuation strain in dielectric elastomers,” J. Polym. Sci. Part B Polym. Phys. 49(7), 504-515 (2011).

[25] Keplinger, C., Li, T., Baumgartner, R., Suo, Z.., Bauer, S., "Harnessing snap-through instability in soft dielectrics to achieve giant voltage-triggered deformation," Soft Matter 8(2), 285 (2012).

[26] Rosset, S., Araromi, O. A.., Shea, H. R., "Maximizing the displacement of compact planar dielectric elastomer actuators," Extrem. Mech. Lett. 3, 72-81, Elsevier Ltd (2015). 